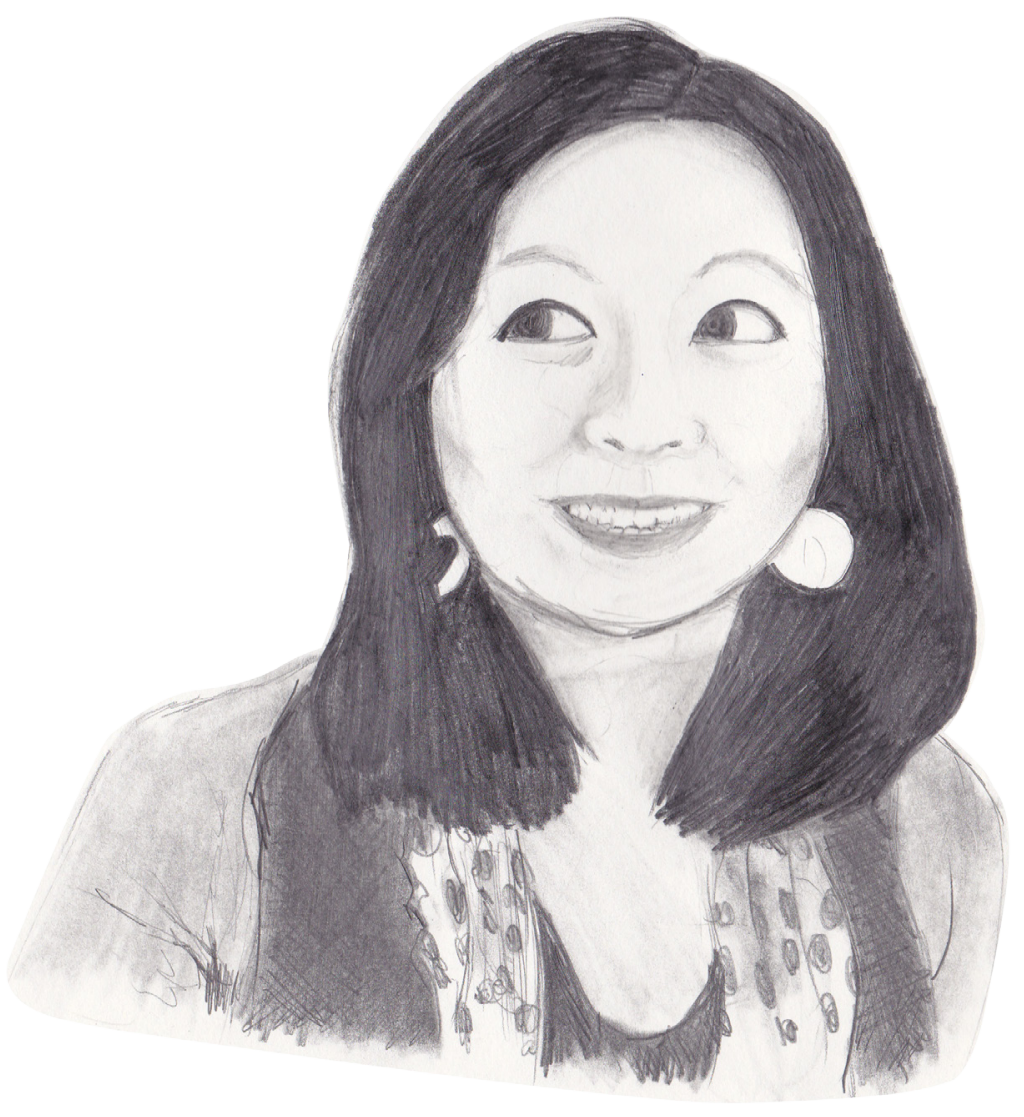

\title{
ZANNAN A. ZHANG
}

Zannan A. Zhang is a senior double majoring in Anthropology and Communication \& Rhetoric at the University of Pittsburgh. She is currently in the throes of applying to graduate school and preparing for functional adulthood. Her greatest academic interests lie in craniofacial development and morphology. Along with a love for the color green, she also exhibits enthusiasm for food, film, and trivia. 
FORBES \& FIFTH

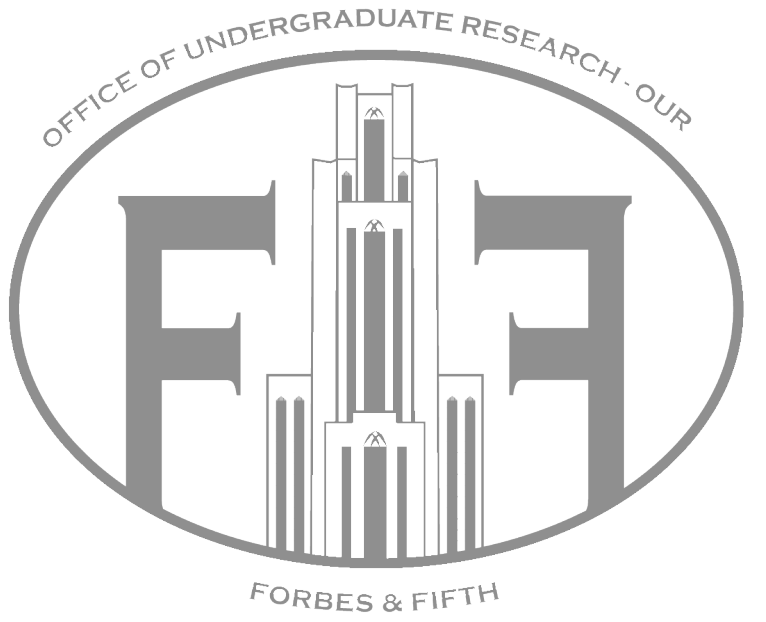




\section{Cross Cultural Considerations for Craniofacial Anomalies}

\section{Introduction}

Craniofacial anomalies have been the subject of a multitude of research studies over the years. The great majority of these studies are primarily concerned with the physical features associated with a medical diagnosis. Two particular anomalies that are often considered are craniosynostosis, the premature fusion of cranial sutures, and palatoschisis (cleft palate), the incomplete formation of the hard palate. The cleft palate anomalies are often grouped with cleft lip malformations of the primary palate. Collectively, these anomalies can be referred to as Craniofacial Anomalies (CFA) and Cleft Lip and Palate (CLP). Of course, the physical aspects of the conditions have been scrutinized with a strong focus on the efficacy of surgical procedures that reduce the abnormally formed facial features and normalize the overall appearance.

The treatment of craniofacial anomalies involves much more than merely the physical aspect. Any condition that has not been commonly accepted by societal standards requires a support team of surgical, psychological, and social work expertise to aid patients in postsurgical adjustments. In more recent years, the psychosocial aspects of CFA and CLP have been under greater scrutiny. Understanding the less essential functional complications for the anomalies can aid in an improved overall quality of life for patients with these conditions.

An important influence on an individual is the home environment, which most directly affects a child's ability to be socialized, since much of social learning has its foundation within the family. What then are the lasting effects of the parental role in the overall result after treatment?

This paper attempts to consider the literature on the confluence of parental involvement in the treatment of CLP and CFA, specifically the underlying cultural understandings that have had the most prevalent influence on the opinion of the conditions. Qualitative research on this subject, though increasing, unfortunately does not address enough of the issues surrounding the social and cultural dynamics that are involved in CFA and CLP treatment. 


\section{General Effects}

Retrospective case studies focusing on self-esteem and social skills have been emerging for adults who have had CFA and CLP. These studies attempt to understand the gender differences and the evolution of treatment methods over an extended period of time. Many of these methods have involved the use of multi-step questionnaires that include both multiple choice scale (1-5 with 1 being not at all effective and 5 being extremely effective) and open ended questions.

Much of the study on the general effects of CFA and CLP on children revolves around the subject of corrective surgery. In Helen Palkes' 1986 article in The Cleft Palate Journal, the focus is on the child's well-being in relation to parental involvement during the process of outpatient care. The paper argues that the purpose of surgery was to secure the "optimal psychological benefit."' The sample group was selected from twenty-four parent-child pairs who were seeking treatment for CFA and CLP at the Washington University Medical Center. ${ }^{2}$ The format of the study included both a questionnaire of demographic information and a Peabody Picture Vocabulary test to assess the intellectual ability of the patients. ${ }^{3}$ The families ranged from single parent families to nuclear families with an assortment of white collar, blue collar, urban, and rural identities. ${ }^{4}$

The attitudes of the parents before surgery were a combination of more negative emotions coupled with lower expressions of parental pride. The most common concerns were over the child's health and well-being. ${ }^{5}$ There was even a sense of a "spread of effect" where other aspects of child's behavior were also viewed negatively. ${ }^{6}$ Children in these families were considered more ill-behaved than their peers, often described as troublemakers. ${ }^{7}$ Also, patients tended to rate themselves as less deformed than when their parents rated them, suggesting that the parents were generally more focused on the anomaly than the children were. ${ }^{8}$ This conclusion was drawn from a drawing test in which both parents and patients were asked to sketch out their families. Parents were more likely to focus on the child's physical differences in their drawings, while the patients were content with just drawing the details of their family dynamic. Overall however, parents were mostly able to predict child's general self esteem, though it was posited that surgery affected parental attitudes towards the child. As the postoperative treat- 
ment progressed, attitudes towards the children were deemed increasingly more positive. ${ }^{9}$ This result was expected as corrective surgery was contingent on parental choice. This conclusion about the strong effect of parental control regarding surgery for CFA and CLP patients was reiterated by a survey of 38 individuals between the ages of 12 and 61 conducted by the National Human Genome Research. ${ }^{10}$ A dominant theme during the surveys was the desire for the patients to convey that the reconstructive surgery methods needed to be more focused on the patient rather than the overall desires of their parents. ${ }^{11}$ In fact, a number of patients struggled with pressure from their parents to continue with more surgeries during their childhood, a sign that some interpreted as a suggestion that their appearance was dissatisfactory to their parents. ${ }^{12}$

The dentist Ronald Strauss was also extremely interested in the perceptions of children with CLP. In 1988, Strauss, along with Hillary Broder and Ronald Helms, conducted a survey of patients with a range of CLP to consider their satisfaction with surgery in regards to their appearance and speech abilities. ${ }^{13}$ They conducted standardized interviews at a cleft palate center to distinguish the difference between perceptions of the adolescent patients and

"A dominant theme during the surveys was the desire for the patients to convey that the reconstructive surgery methods needed to be more focused on the patient rather than the overall desires of their parents." perceptions of their parents. ${ }^{14}$ It seemed that patients tended to be generally pleased with their appearance and speech ability, but many initially entered treatment with decidedly low expectations due to past experiences with surgery. ${ }^{15}$ There were no clear gender differences between patient responses, and the parental responses tended to align well with the responses of the patients. ${ }^{16}$ This new study demonstrated that parents and children were in agreement during the treatment period, a conclusion that is not as widely reported in other studies.

In 1991, Strauss and Broder continued to focus on the general issues with psychosocial research when applied to CLP and CFA. They stated that there needed to be a better inclusion of "the complexity of factors and interaction effects that can affect psychological function [which] must be considered in research design." 17 Their suggestions included the use of matched control groups and comparison groups, rather 
than relying on one set of researchers' interpretations of interviews. ${ }^{18}$ There was also an emphasis placed on better cultural understanding and a need for a broader look at the conditions rather than merely including single treatment centers. ${ }^{19}$

This interest in parental attitudes persisted for a few years as Mary Pannbacker and Jane Scheurle conducted another survey in 1993, including multiple regions within the United States. ${ }^{20}$ Both researchers agreed that parental involvement was needed to complete a comprehensive team treatment for children with CLP, and that therefore, more research was needed regarding the effect of parental attitudes toward their children's medical treatment. ${ }^{21}$

More recently in 2012, a nationwide Australian survey assessed the quality of life of CLP patients against population norms. The study, led by Peter Foo, warned that there needed to be more time allotted to properly understand the results of the survey, but that the cross-sectional prospective survey did yield some interesting results. ${ }^{22}$ The self-report style of the questionnaire concluded that the overall quality of life was considered very satisfactory, save for the physical transformation which was often deemed unsatisfactory or in need of improvement. ${ }^{23}$ This article once again reemphasized the theme of physical appearance being a primary concern of CLP patients.

Regarding the general thoughts on the psychosocial aspects of CLP and CFA, the literature on the subject appears to be moving towards a goal of more positive social outcomes for patients rather than just the appearance. In the 1998 Turner, Rumsey, and Sandy article for the European Journal of Orthodontics, the belief was that parental and patient perceptions frequently overlooked in favor of the general physical health of the patient. ${ }^{24}$ Turner argued that because the birth of a baby with CLP is rarely predicted before birth, families of children with anomalies tended to report higher personal stress and familial conflict while adjusting to the news. ${ }^{25}$ This rather easily predictable outcome often resulted in children with CLP exhibiting shy and sometimes socially inhibited behavior, the source of which, in some cases, was the parents giving CLP children more room for misbehavior by adjusting their leniency. ${ }^{26}$ A similar conclusion was made in 2013 by a research group from the National Institute of Health. The comparative review drew on previously conducted studies that focused on the effectiveness 
of physical and psychological care of patients with CLP. ${ }^{27}$ Conclusions were difficult as the authors believed there was a paucity of evidence to support any consistent long term effects on various patients.

Research by Alice Pope and Heather Snyder suggested that the effects on CLP and CFA children's behavior may have been somewhat exaggerated. ${ }^{28}$ Their examination of CFA patients in an urban setting used clinical description rather than utilizing a survey or questionnaire that directly involved patient responses and concluded that, though CFA patients may have a few social skill deficits, they were equally as likely as other children to have elevated rates of competency in other social areas. In fact, CFA patients often scored higher in their sense of self and determination.

\section{Cultural Influences}

Culture has an extremely broad definition that includes the status of social norms for a group of people who share similar identities. There have been cultural anthropological studies on both western and nonwestern groups of people on the influence of long held cultural beliefs on the treatment of children with CFA and CLP.

In 1985, Ronald Strauss considered the difference between Western medicine and other international case studies of CLP and CFA. He urged for a response to birth defects in their cultural and social context, raising the ethical questions of whether or not surgical correction for CFA should be routine after birth. ${ }^{29}$ Strauss described two distinct non-western cultures in Israel and Nigeria to illustrate the complexity of cultural influence on CFA treatment.

Israel represented a developed multicultural nation composed of a primarily Jewish (western and non-western) and Arab population. The variation in culture led to two similar views on CFA, which Strauss labeled as either fatalistic or fixing. Strauss argued that the non-western Jews took a more fatalistic view regarding CFA and speculated about a curse on the family, leading to a passive stance towards treatment. ${ }^{30}$ On the other hand, the European Jews took a very active view of the situation and elected for surgery due to the "value placed on normalization and social integration." ${ }^{31}$ The largely Muslim Arab people were also pinned with a fatalistic spiritual attitude. Strauss did also mention that the views on surgery in Israel are also strongly influenced by the avail- 
ability and accessibility of surgery in Israel. ${ }^{32}$

Nigeria was presented as an example of a developing nation in West Africa, composed of two-hundred fifty tribal groups. In Nigeria,

"In Nigeria, due to the fact that infectious diseases receive most of the medical attention, a minor physical handicap such as CLP or CFA was sure to lead to poverty. Thus, a 'disfigured child [is] held to represent the family's social position and is regarded with shame.' These physical handicaps were linked to witchcraft and led

to concealment or neglect."

due to the fact that infectious diseases receive most of the medical attention, a minor physical handicap such as CLP or CFA was sure to lead to poverty. ${ }^{33}$ Thus, a "disfigured child [is] held to represent the family's social position and is regarded with shame." ${ }^{34}$ These physical handicaps were linked to witchcraft and led to concealment or neglect. ${ }^{35}$ However, much of the treatment of CLP and CFA children was hidden and shrouded from outsiders to perhaps hide the true implications of having an unaccepted child in a poorer society. ${ }^{36}$

Yet, later research into CLP and CFA cross-cultural treatment attempted a more varied and perhaps more fatalistic view of the circumstances. Nancy Scheper-Hughes in 1990 brought in the concept of ethno-eugenic survivalism by consolidating various ethnographies. She argued that the heroic "saving" of CLP and CFA children ought to be thoroughly scrutinized within a cultural context. ${ }^{37}$ Because many environments were unfavorable to infant survival, congenital anomalies have long been "the primary rationale for infanticide world-wide." 38 These "untouchables," or physically different doomed infants, were often thought of as supernatural or non-human. ${ }^{39}$ Sometimes infants were set out to die in order to protect others from being polluted, as was observed in the Nuer people of Central Africa, who regarded the deformed child as a mistake. ${ }^{40}$ Scheper-Hughes also gave the example of the rural Irish in West Kerry who believed in changelings, or evil creatures brought to replace newborns in the night. ${ }^{41}$ These children were exposed and burned throughout the years..$^{42}$ Ethnographies of the Bariba people of Benin called these children witches, witch infants, and throw-away children. ${ }^{43}$ Scheper-Hughes argued that these third world area interpretations considered widespread infanticide to be a humane way of controlling the population. ${ }^{44}$ Yet, she was also quick to point 
out that those who survived their CLP and CFA conditions were also regarded as exemplary individuals avenging fate by surviving. ${ }^{45}$

In response to ScheperHughes's somewhat dramatic conclusion, Robert J. Shprintzen explained that the treatment methods for CFA and CLP were not as homogenous as Scheper-Hughes

"Because many environments were unfavorable to infant survival, congenital anomalies have long been 'the primary rationale for infanticide world-wide.' These 'untouchables,' or physically different doomed infants, were often thought of as supernatural or non-human." assumed. Rather, the conclusion was that these cultural variations ought to be taken into account and assessed by a group that specializes in cross-cultural communication in order to deliver the best treatment for the patient, rather than taking a hands-off approach to the matter.

Still, viewing CFA and CLP through distinct cultural lenses remains a challenging task. Qualitative interviews have been conducted more recently in South Africa where the convergence of Western healthcare and traditional healing methods has led to a better understanding of the negative social reactions due to persistent cultural myths. ${ }^{46}$ These interviews once again pointed to an ability of affected patients to overcome their anomaly by becoming more philosophical and adventurous.

A more structured approach to examining the cultural effect on the family environment of CLP patients emerged in 2011 in a study by John Loh and Micol Ascoli, two psychiatrists concerned with the providing an cross-cultural explanatory model for reactions to children with CLP. The demographics included the Chinese, Africans, and Indians. The psychological profiles of adolescent and adult individuals with CLP were largely high for their self-esteem scores. ${ }^{47}$ This high selfesteem was attributed to the traditional Chinese culture's emphasis on interpersonal relationships and social harmony. ${ }^{48}$ The parental attitude of the Chinese was referred to as more accepting of the possibility of emotional instability for their children. ${ }^{49}$ That is not to say that the CLP children were considered elevated in any way. Normality was still the main desire for the Chinese study group. Attitudes toward CLP in Africa was considered more severe as the incidence probability was somewhat lower compared to the West and Asia. ${ }^{50}$ The explanation was once again considered more supernatural and linked with witchcraft, imbalance, 
and impurity, a largely religiously biased approach to the anomaly. ${ }^{51}$ The Indian individuals were also dealing with the concept of curses, evil spirits, and retribution for past sins. The anxiety in social interaction was high, but this anxiety was more likely the cause of familial isolation for protection of the child. ${ }^{52}$ Yet, this article concluded that the literature regarding these cross-cultural attitudes still suffered from severe limitations on the information needed for these studies. Comparative studies on varying traditional treatment methods was also scarce but deemed a high possibility by the authors.

More recently in 2013, a research group in Hyderabad, India published an article on the perceptions of family members in the rural outskirts of that community. The commonly held belief in that region of

"The commonly held belief in [Hyerabad,] India, explains the presence of a CLP as the result of an eclipse, a superstition that often leads to neutrality towards the welfare of the child. [...] The article once again pointed to the gap in the craniofacial anomaly literature between cultural practices and clinical practices which can inhibit proper medical care." India explained the presence of a CLP as the result of an eclipse, a superstition that often leads to neutrality towards the welfare of the child. ${ }^{53}$ The authors of this piece concluded that the physicians in the area needed to design an educational protocol for the community to better understand how to care for patients with these malformations. ${ }^{54}$ The article once again pointed to the gap in the craniofacial anomaly literature between cultural practices and clinical practices which can inhibit proper medical care.

\section{Future Considerations}

There is no perfect answer to the question of what parental and familial influences have on a child, but the accepted conclusion is generally along the lines of the predictable. Positive familial environments and highly involved parents produce more well-adjusted children. However, research and common sense both point to the plethora of random and ambiguous situations that cause answers to not lie on a simple "extremely well-adjusted" and "extremely not well-adjusted" binary scale of comparison.

Every few years, a call is made for more research on the subject 
of improving qualitative methods of research, and the issues remain constant. There is an understanding that cross cultural problems are present, but cultural anthropological research on the subject is hardly consolidated. The regional distribution is scattered. Overall, there is much room for more research on this subject, including newer and more accurate styles of surveying, interviewing, and a better method of comparison between differing groups of individuals. 
FORBES \& FIFTH 